Proceedings of the Institute of Mathematics and Mechanics,

National Academy of Sciences of Azerbaijan

Volume 47, Number 1, 2021, Pages 67-77

https://doi.org/10.30546/2409-4994.47.1.67

\title{
PARA-COMPLEX STRUCTURES ON LINEAR COFRAME BUNDLE WITH SASAKIAN METRIC
}

\author{
HABIL FATTAYEV
}

\begin{abstract}
By using a Riemannian metric on a differentiable manifold, the Sasakian metric is introduced on the linear coframe bundle of the Riemannian manifold. Geometric properties of Levi-Civita connection of Sasakian metric are investigated. Also, para-complex structures on the linear coframe bundle with Sasakian metric are constructed and some interesting properties of those structures are studied.
\end{abstract}

\section{Introduction}

Let $M$ be an $n$-dimensional manifold of class $C^{\infty}$. The problem of extending differential-geometrical structures on $M$ to its fiber bundles has been the subject of a number of papers. An account of these can be found in Yano and Ishihara [22] (see, also [2]). In [21], Sasaki by using a Riemannian metric $g$ on a differentiable manifold $M$, constructed a Riemannian metric $\tilde{g}$ on the tangent bundle $T(M)$ of $M$. Then, some geometers such as Kowalski, Aso, Musso and Tricerri studied interesting geometric properties of this metric, that is called Sasaki metric (see [1], [8], [11]). Some properties and applications for the Riemannian metrics of the cotangent, linear frame, linear coframe and tensor bundles are given in $[3,5,9,10$, 16-18]. Also noteworthy are the papers devoted to the study of various differential geometric structures, including submanifolds of Sasakian manifolds. V.A.Khan and M.A.Khan studied pseudo-slant submanifolds of Sasakian manifolds (see [7]). The main results related to the projective curvature tensor in Sasakian manifolds are due to U.K.Gautam, A.Haseeb and R.Prasad (see [6]).

Let $M_{2 k}$ be a $2 k$-dimensional differentiable manifold endowed with an almost (para) complex structure $\varphi$ and a pseudo-Riemannian metric $g$ of signature $(k, k)$ such that $g(\varphi X, Y)=g(X, \varphi Y)$ for arbitrary vector fields $X$ and $Y$ on $M_{2 k}$, i.e. $g$ is pure with respect to $\varphi$. The metric $g$ is called Nordenian metric. Nordenian metrics are also referred to as anti-Hermitian metrics or B-metrics. They present extensive application in mathematics as well as in theoretical physics. Many authors considered almost (para) complex Nordenian structures on the tangent, cotangent and tensor bundles [4, 12-15].

2010 Mathematics Subject Classification. 55R10, 53C07, 55C15.

Key words and phrases. Riemannian manifold, linear coframe bundle, Sasakian metric, paracomplex structure, almost para-Nordenian structure. 
This paper is devoted to the investigation of para-Nordenian structures in the linear coframe bundle with Sasakian metric. In 2 we briefly describe the definitions and results that are needed later, after which the Sasakian metric ${ }^{S} g$ and the Levi-Civita connection ${ }^{S} \nabla$ of this metric in the linear coframe bundle $F^{*}(M)$ over a Riemannian manifold $(M, g)$ are studied in 3 . The para-Nordenian structures on the linear coframe bundle $F^{*}(M)$ with Sasakian metric are introduced in 4 . In 5 we study the almost para-holomorphic vector fields on the linear coframe bundle $F^{*}(M)$ with Sasakian metric. The integrabilities of almost para-Nordenian structures on the $F^{*}(M)$ with Sasakian metric are investigated in 6 .

\section{Preliminaries}

In this section we shall summarize briefly the basic definitions and results which be used later. Let $(M, g)$ be an $n$-dimensional Riemannian manifold and $F^{*}(M)$ its coframe bundle (see, $[19,20])$. The coframe bundle $F^{*}(M)$ over $M$ consists of all pairs $\left(x, u^{*}\right)$, where $x$ is a point of $M$ and $u^{*}$ is a basis (coframe) for the cotangent space $T_{x}^{*} M$. We denote by $\pi$ the natural projection of $F^{*}(M)$ to $M$ defined by $\pi\left(x, u^{*}\right)=x$. If $\left(U ; x^{1}, x^{2}, \ldots, x^{n}\right)$ is a system of local coordinates in $M$, then a coframe $u^{*}=\left(X^{\alpha}\right)=\left(X^{1}, X^{2}, \ldots, X^{n}\right)$ for $T_{x}^{*} M$ can be expressed uniquely in the form $X^{\alpha}=X_{i}^{\alpha}\left(d x^{i}\right)_{x}$ and hence

$$
\left(\pi^{-1}(U) ; x^{1}, x^{2}, \ldots, x^{n}, X_{1}^{1}, X_{2}^{1}, \ldots, X_{n}^{n}\right)
$$

is a system of local coordinates in $F^{*}(M)$ (see, [19]). Indices $i, j, k, \ldots, \alpha, \beta, \gamma, \ldots$ have range in $\{1,2, \ldots, n\}$, while indices $A, B, C, \ldots$ have range in

$$
\left\{1, \ldots, n, n+1, \ldots, n+n^{2}\right\} .
$$

We put $h_{\alpha}=\alpha \cdot n+h$. Summation over repeated indices is always implied.

We denote by $\Im_{s}^{r}(M)$ the set of all differentiable tensor fields of type $(r, s)$ on $M$. Let $V=V^{i} \partial_{i}$ and $\omega=\omega_{i} d x^{i}$ be the local expressions in $U \subset M$ of a vector and a covector (1-form) fields $V \in \Im_{0}^{1}(M)$ and $\omega \in \Im_{1}^{0}(M)$, respectively. Then the complete and horizontal lifts ${ }^{C} V,{ }^{H} V \in \Im_{0}^{1}\left(F^{*}(M)\right)$ of $V$ and the $\beta$-th vertical lifts $V_{\beta} \omega \in \Im_{0}^{1}\left(F^{*}(M)\right)(\beta=1,2, \ldots, n)$ of $\omega$ are given, respectively, by

$$
{ }^{C} V=\left(\begin{array}{c}
V^{i} \\
-X_{j}^{\alpha} \partial_{i} V^{j}
\end{array}\right), \quad{ }^{H} V=\left(\begin{array}{c}
V^{i} \\
X_{j}^{\alpha} \Gamma_{i k}^{j} V^{k}
\end{array}\right), \quad{ }^{V_{\beta}} \omega=\left(\begin{array}{c}
0 \\
\delta_{\beta}^{\alpha} \omega_{i}
\end{array}\right)
$$

with respect to the natural frame $\left\{\partial_{i}, \partial_{i_{\alpha}}\right\}=\left\{\frac{\partial}{\partial x^{i}}, \frac{\partial}{\partial X_{i}^{\alpha}}\right\}$, (see [10] for more details).

The vertical lift of a smooth function $f$ on $M$ is a function ${ }^{V} f$ on $F^{*}(M)$ defined by $V f=f \circ \pi$.

Let $\left(U, x^{i}\right)$ be a coordinate system in $M$. In $U \in M$, we put

$$
X_{(i)}=\frac{\partial}{\partial x^{i}}, \theta^{(i)}=d x^{i}, i=1,2, \ldots, n .
$$

Taking account of (2.1), we easily see that the components of ${ }^{H} X_{(i)}$ and ${ }^{V_{\alpha}} \theta^{(i)}$ are respectively, given by

$$
D_{i}={ }^{H} X_{(i)}=\left(A_{i}^{H}\right)=\left(\begin{array}{c}
\delta_{i}^{h} \\
X_{j}^{\alpha} \Gamma_{i h}^{j}
\end{array}\right),
$$




$$
D_{i_{\alpha}}={ }^{V_{\alpha}} \theta^{(i)}=\left(A_{i_{\alpha}}^{H}\right)=\left(\begin{array}{c}
0 \\
\delta_{\beta}^{\alpha} \delta_{h}^{i}
\end{array}\right)
$$

with respect to the natural frame $\left\{\partial_{i}, \partial_{i_{\alpha}}\right\}$. We call the set $\left\{{ }^{H} X_{(i)},{ }^{V_{\alpha}} \theta^{(i)}\right\}$ the frame adapted to the Levi-Civita connection $\nabla_{g}$. On putting

$$
D_{i}={ }^{H} X_{(i)}, \quad D_{i_{\alpha}}={ }^{V_{\alpha}} \theta^{(i)},
$$

we write the adapted frame as $\left\{D_{I}\right\}=\left\{D_{i}, D_{i_{\alpha}}\right\}$. From equations (2.2), (2.3), and (2.1) we see that ${ }^{H} V$ and ${ }^{V_{\alpha}} \omega$ have respectively, components

$$
\begin{gathered}
{ }^{H} V=V^{i} D_{i},{ }^{H} V=\left({ }^{H} V^{I}\right)=\left(\begin{array}{c}
V^{i} \\
0
\end{array}\right), \\
{ }^{V_{\alpha}} \omega=\sum_{i} \omega_{i} \delta_{\alpha}^{\beta} D_{i_{\alpha}},{ }^{V_{\alpha}} \omega=\left({ }^{V_{\alpha}} \omega^{I}\right)=\left(\begin{array}{c}
0 \\
\delta_{\alpha}^{\beta} \omega_{i}
\end{array}\right)
\end{gathered}
$$

with respect to the adapted frame $\left\{D_{I}\right\}$, where $V^{i}$ and $\omega_{i}$ being local components of $V \in \Im_{0}^{1}(M)$ and $\omega \in \Im_{1}^{0}(M)$, respectively.

For each $x \in M$, the scalar product $g^{-1}=\left(g^{i j}\right)$ is defined on the cotangent space $T_{x}^{*} M$ by $g^{-1}(\omega, \theta)=g^{i j} \omega_{i} \theta_{j}$ for all $\omega, \theta \in \Im_{1}^{0}(M)$.

The bracket operation of vertical and horizontal vector fields is given by the formulas

$$
\begin{gathered}
{\left[{ }^{V_{\beta}} \omega,{ }^{V_{\gamma}} \theta\right]=0,\left[{ }^{H} X,{ }^{V_{\beta}} \omega\right]={ }^{V_{\beta}}\left(\nabla_{X} \omega\right),} \\
{\left[{ }^{H} X,{ }^{H} Y\right]={ }^{H}[X, Y]+\sum_{\sigma=1}^{n}{ }^{V_{\sigma}}\left(X^{\sigma} \circ R(X, Y)\right)}
\end{gathered}
$$

for all $X, Y \in \Im_{0}^{1}(M)$ and $\omega, \theta \in \Im_{1}^{0}(M)$, where $R$ denotes the curvature tensor field of the linear connection $\nabla$.

\section{The Sasakian metric on the coframe bundle}

We define a Riemannian metric ${ }^{S} g$ on the coframe bundle $F^{*}(M)$ by the following three equations

$$
\begin{gathered}
{ }^{S} g\left({ }^{H} X,{ }^{H} Y\right)={ }^{V}(g(X, Y))=g(X, Y) \circ \pi, \\
{ }^{S} g\left({ }^{H} X,{ }^{V_{\beta}} \omega\right)=0, \\
{ }^{S} g\left({ }^{V_{\beta}} \omega,{ }^{V_{\gamma}} \theta\right)=\delta^{\beta \gamma}{ }\left(g^{-1}(\omega, \theta)\right)=\delta^{\beta \gamma}\left(g^{-1}(\omega, \theta) \circ \pi\right)
\end{gathered}
$$

for any $X, Y \in \Im_{0}^{1}(M)$ and $\omega, \theta \in \Im_{1}^{0}(M)$ (see, [5]). We call the metric ${ }^{S} g$ the Sasakian metric on the coframe bundle $F^{*}(M)$ over the Riemannian manifold $(M, g)$. Since any tensor field of type $(0,2)$ on the $F^{*}(M)$ is completely determined by its action on vector fields of type ${ }^{H} X$ and ${ }^{V_{\beta}} \omega$ it follows that ${ }^{S} g$ is completely determined by its eqs (3.1), (3.2) and (3.3). The metric ${ }^{S} g$ is a Riemannian metric on $F^{*}(M)$ uniquely determined by the metric $g$.

From equations (3.1), (3.2) and (3.3) it follows that

$$
\begin{gathered}
{ }^{S} g_{i j}={ }^{S} g\left(D_{i}, D_{j}\right)={ }^{V}\left(g\left(\partial_{i}, \partial_{j}\right)\right)=g_{i j}, \\
{ }^{S} g_{i_{\alpha} j}={ }^{S} g\left(D_{i_{\alpha}}, D_{j}\right)=0, \\
{ }^{S} g_{i_{\alpha} j_{\beta}}={ }^{S} g\left(D_{i_{\alpha}}, D_{j_{\beta}}\right)=\delta^{\alpha \beta V}\left(g^{-1}\left(d x^{i}, d x^{j}\right)\right)=\delta^{\alpha \beta} g^{i j},
\end{gathered}
$$


i.e. ${ }^{S} g$ has components in the form

$$
S_{g}=\left(\begin{array}{cc}
g_{i j} & 0 \\
0 & \delta^{\alpha \beta} g^{i j}
\end{array}\right)
$$

with respect to the adapted frame $\left\{D_{I}\right\}$.

Let us consider local 1 -forms $\tilde{\eta}^{I}$ in $\pi^{-1}(U)$ defined by

$$
\tilde{\eta}^{I}=\bar{A}^{I}{ }_{J} d x^{J}
$$

where

$$
A^{-1}=\left(\begin{array}{ll}
\bar{A}^{I} & J
\end{array}\right)=\left(\begin{array}{cc}
\bar{A}_{j}^{i} & \bar{A}_{j_{\beta}}^{i} \\
\bar{A}_{j}^{i_{\alpha}} & \bar{A}_{j_{\beta}}^{i_{\alpha}}
\end{array}\right)=\left(\begin{array}{cc}
\delta_{j}^{i} & 0 \\
-X_{m}^{\alpha} \Gamma_{i j}^{m} & \delta_{\beta}^{\alpha} \delta_{i}^{j}
\end{array}\right) .
$$

The matrix (3.8) is the inverse of the matrix

$$
A=\left(A_{K}{ }^{J}\right)=\left(\begin{array}{cc}
A_{k}^{j} & A_{k_{\gamma}}^{j} \\
A_{k}^{j_{\beta}} & A_{k_{\gamma}}^{j_{\beta}}
\end{array}\right)=\left(\begin{array}{cc}
\delta_{k}^{j} & 0 \\
X_{m}^{\beta} \Gamma_{j k}^{m} & \delta_{\gamma}^{\beta} \delta_{j}^{k}
\end{array}\right)
$$

of the transformation $D_{K}=A_{K}{ }^{J} \partial_{J}$ ( see (2.2) and (2.3)). It is easy to establish that the set $\left\{\tilde{\eta}^{I}\right\}$ is the coframe dual to the adapted frame $\left\{D_{K}\right\}$, i.e.

$$
\tilde{\eta}^{I}\left(D_{K}\right)=\bar{A}^{I}{ }_{J} A_{K}{ }^{J}=\delta_{K}^{I} .
$$

Since the adapted frame is non-holonomic, we put

$$
\left[D_{I}, D_{J}\right]=\Omega_{I J}^{K} D_{K}
$$

from which we have

$$
\Omega_{I J}^{K}=\left(D_{I} A_{J}^{L}-D_{J} A_{I}^{L}\right) \bar{A}_{L}^{K} .
$$

According to (2.2), (2.3), (3.5) and (3.6), the components of non-holonomic object $\Omega_{I J}{ }^{K}$ are given by

$$
\left\{\begin{array}{l}
\Omega_{i j_{\beta}{ }^{k}}{ }^{k_{\gamma}}=-\Omega_{j_{\beta}{ }^{i}{ }^{k_{\gamma}}}=-\delta_{\beta}^{\gamma} \Gamma_{i k}^{j}, \\
\Omega_{i j}{ }^{k_{\gamma}}=X_{m}^{\gamma} R_{i j k}^{m}
\end{array}\right.
$$

all the others being zero, where $R_{i j k}^{m}$ local components of the curvature tensor field $R$ of $\nabla_{g}$.

Let ${ }^{S} \nabla$ be the Levi-Civita connection determined by the Sasakian metric ${ }^{S} g$ on the coframe bundle $F^{*}(M)$. We put

$$
{ }^{S} \nabla_{D_{I}} D_{J}={ }^{S} \Gamma_{I J}^{K} D_{K}
$$

From the equation

$$
{ }^{S} \nabla_{X} Y-{ }^{S} \nabla_{Y} X=[X, Y], \forall X, Y \in \Im_{0}^{1}\left(F^{*}(M)\right)
$$

we have

The equation

$$
{ }^{S} \Gamma_{I J}^{K}-{ }^{S} \Gamma_{J I}^{K}=\Omega_{I J}{ }^{K}
$$

has form

$$
\left({ }^{S} \nabla_{X}{ }^{S} g\right)(Y, Z)=0
$$

$$
D_{L}{ }^{S} g_{I J}-{ }^{S} \Gamma_{L I}^{K}{ }^{S} g_{K J}-{ }^{S} \Gamma_{L J}^{K}{ }^{S} g_{I K}=0
$$

with respect to the adapted frame $\left\{D_{K}\right\}$. By using (3.8) and (3.9), we obtain:

$$
\begin{aligned}
& { }^{S} \Gamma_{I J}^{K}=\frac{1}{2}{ }^{S} g^{K L}\left(D_{I}{ }^{S} g_{L J}+D_{J}{ }^{S} g_{I L}-D_{L}{ }^{S} g_{I J}\right)+ \\
& +\frac{1}{2}\left(\Omega_{I J}{ }^{K}+\Omega^{K}{ }_{I J}+\Omega^{K}{ }_{J I}\right) \text {, }
\end{aligned}
$$


where $\Omega_{I J}^{K}={ }^{S} g^{K L S} g_{P J} \Omega_{L I}^{P}$ and

$$
\left({ }^{S} g\right)^{-1}=\left({ }^{S} g^{K J}\right)=\left(\begin{array}{cc}
g^{k j} & 0 \\
0 & \delta_{\gamma \beta} g_{k j}
\end{array}\right) .
$$

Taking account (3.4), (3.7) and (3.11), we obtain from (3.10)

$$
\begin{cases}{ }^{S} \Gamma_{i j}^{k}=\Gamma_{i j}^{k}, & { }^{S} \Gamma_{i_{\alpha} j_{\beta}}^{k}={ }^{S} \Gamma_{i_{\alpha} j}^{k_{\gamma}}={ }^{S} \Gamma_{i_{\alpha} j_{\beta}}^{k_{\gamma}}=0, \\ { }^{S} \Gamma_{i j_{\beta}}^{k}=\frac{1}{2} X_{m}^{\beta} R_{\cdot i}^{k j m}, & { }^{S} \Gamma_{i_{\alpha} j}^{k}=\frac{1}{2} X_{m}^{\alpha} R_{\cdot j}^{k} \cdot{ }^{i m}, \\ { }^{S} \Gamma_{i j}^{k_{\gamma}}=\frac{1}{2} X_{m}^{\gamma} R_{i j k}^{m}, & { }^{S} \Gamma_{i j_{\beta}}^{k_{\gamma}}=-\delta_{\gamma}^{\beta} \Gamma_{i k}^{j} .\end{cases}
$$

Let $\tilde{X}, \tilde{Y} \in \Im_{0}^{1}\left(F^{*}(M)\right)$ and $\tilde{X}=\tilde{X}^{I} D_{I}, \tilde{Y}=\tilde{Y}^{J} D_{J}$. Then the covariant derivative ${ }^{S} \nabla_{\tilde{Y}} \tilde{X}$ along $\tilde{Y}$ has components in the form

$$
{ }^{S} \nabla_{\tilde{Y}} \tilde{X}^{I}=\tilde{Y}^{J} D_{J} \tilde{X}^{I}+{ }^{S} \Gamma_{J K}^{I} \tilde{X}^{K} \tilde{Y}^{J}
$$

with respect to the adapted frame $\left\{D_{I}\right\}$.

Using (2.4)-(2.6), (3.1)-(3.3), (3.12) and (3.13), we have

Theorem 3.1. Let $M$ be a Riemannian manifold with metric $g$ and ${ }^{S} \nabla$ be the Levi-Civita connection of the coframe bundle $F^{*}(M)$ equipped with Sasakian metric ${ }^{S} g$. Then ${ }^{S} \nabla$ satisfies

$$
\begin{gathered}
i)^{S} \nabla_{V_{\alpha} \omega}{ }^{V_{\beta}} \theta=0, \\
i i)^{S} \nabla_{V_{\alpha} \omega}{ }^{H} Y=\frac{1}{2}^{H}\left(R\left(\tilde{X}^{\alpha}, \tilde{\omega}\right) Y\right), \\
i i i)^{S} \nabla_{{ }^{H} X}{ }^{V_{\beta}} \theta={ }^{V_{\beta}}\left(\nabla_{X} \theta\right)+\frac{1}{2}{ }^{H}\left(R\left(\tilde{X}^{\beta}, \tilde{\theta}\right) X\right), \\
i v)^{S} \nabla^{H}{ }^{H} Y={ }^{H}\left(\nabla_{X} Y\right)+\frac{1}{2} \sum_{\sigma=1}^{n}{ }^{V_{\sigma}}\left(X^{\sigma} \circ R(X, Y)\right)
\end{gathered}
$$

for all $X, Y \in \Im_{0}^{1}(M)$ and $\omega, \theta \in \Im_{1}^{0}(M)$, where $\tilde{\omega}=g^{-1} \circ \omega \in \Im_{0}^{1}(M)$, $\tilde{\theta}=g^{-1} \circ \theta \in \Im_{0}^{1}(M), \tilde{X}^{\alpha}=g^{-1} \circ X^{\alpha} \in \Im_{0}^{1}(M)$.

We note that the analogue of Theorem 3.1 in the case of cotangent bundle is proved in [18].

\section{Para-Nordenian structures on $\left.\left(F^{*}(M),{ }^{S} g\right)\right)$}

An almost para-complex manifold is an almost product manifold $\left(M_{n}, \varphi\right)$, $\varphi^{2}=I$, such that the two eigenbundles $T^{+}\left(M_{n}\right)$ and $T^{-}\left(M_{n}\right)$ associated to the two eigenvalues +1 and -1 of $\varphi$, respectively, have the same rank. The dimension of an almost paracomplex manifold is necessarily even.

A tensor field $t \in \Im_{q}^{0}\left(M_{2 n}\right)$ is said to be a pure with respect to the para-complex structure $\varphi$, if

$$
t\left(\varphi X_{1}, X_{2}, \ldots, X_{q}\right)=t\left(X_{1}, \varphi X_{2}, \ldots, X_{q}\right)=t\left(X_{1}, X_{2}, \ldots, \varphi X_{q}\right)
$$

for any $X_{1}, X_{2}, \ldots, X_{q} \in \Im_{0}^{1}\left(M_{2 n}\right)$.

We define the following operator $\phi_{\varphi}$ associated with $\varphi$ and apply to the pure tensor field $t$ :

$$
\left(\phi_{\varphi} t\right)\left(Y, X_{1}, X_{2}, \ldots, X_{q}\right)=(\varphi Y)\left(t\left(X_{1}, X_{2}, \ldots, X_{q}\right)\right)
$$




$$
\begin{gathered}
\left.-Y\left(t\left(\varphi X_{1}, X_{2}, \ldots, X_{q}\right)\right)+t\left(\left(L_{X_{1}} \varphi\right) Y, X_{2}, \ldots, X_{q}\right)\right) \\
+\cdots+t\left(X_{1}, X_{2}, \ldots,\left(L_{X_{q}} \varphi\right) Y\right) .
\end{gathered}
$$

We note that $\phi_{\varphi} t \in \Im_{q}^{0}\left(M_{2 n}\right)$. If $\phi_{\varphi} t=0$ then $t$ is said to be an almost paraholomorphic (see $[5,15,16,18]$ ).

Definition 4.1. In a manifold with almost para-complex structure $\varphi$, a vector field $X$ is called an almost para-holomorphic vector field if $L_{X} \varphi=0$.

A Riemannian manifold $\left(M_{2 n}, g\right)$ with an almost para-complex structure $\varphi$ is said to be almost para-Nordenian if the Riemannian metric $g$ is pure with respect to $\varphi$. It is well known that the almost para-Nordenian manifold is para-Kahler $\left(\nabla_{g} \varphi=0\right)$ if and only if $g$ is para-holomorphic $\left(\phi_{\varphi} g=0\right)$ (see [18]).

Let $\left(F^{*}(M),{ }^{S} g\right)$ be the linear coframe bundle with the Sasakian metric ${ }^{S} g$. Define a tensor field $F_{\alpha}$ of type $(1,1)$ on $F^{*}(M)$ for each $\alpha=12, \ldots, n$, by

$$
F_{\alpha}\left({ }^{H} X\right)={ }^{V_{\alpha}} \tilde{X}, \quad F_{\alpha}\left({ }^{V_{\beta}} \omega\right)=\delta_{\alpha}^{\beta H} \tilde{\omega}
$$

for any $X \in \Im_{0}^{1}(M)$ and $\omega \in \Im_{1}^{0}(M)$, where $\tilde{X}=g \circ X \in \Im_{1}^{0}(M), \tilde{\omega}=g^{-1} \circ \omega \in$ $\Im_{0}^{1}(M)$ and the horizontal lifts are considered with respect to the Levi-Civita connection of $g$. Each $F_{\alpha}$ satisfies the condition

$$
F_{\alpha}^{2}=I
$$

Indeed, by virtue of (4.1), we have

$$
\begin{aligned}
& F_{\alpha}^{2}\left({ }^{H} X\right)=F_{\alpha}\left(F_{\alpha}\left({ }^{H} X\right)\right)=F_{\alpha}\left({ }^{{ }{ }^{\alpha}} \tilde{X}\right)=\delta_{\alpha}^{\alpha H} \tilde{\tilde{X}}={ }^{H} X, \\
& F_{\alpha}^{2}\left({ }^{V_{\beta}} \omega\right)=F_{\alpha}\left(F_{\alpha}\left({ }^{V_{\beta}} \omega\right)\right)=F_{\alpha}\left(\delta_{\alpha}^{\beta H} \tilde{\omega}\right)=\delta_{\alpha}^{\beta V_{\alpha}} \tilde{\tilde{\omega}}={ }^{V_{\beta}} \omega
\end{aligned}
$$

for any $X \in \Im_{0}^{1}(M)$ and $\omega \in \Im_{1}^{0}(M)$, which implies $F_{\alpha}^{2}=I$ for each $\alpha=12, \ldots, n$.

The following theorem holds.

Theorem 4.1. The triple $\left(F^{*}(M),{ }^{S} g, F_{\alpha}\right)$, for each $\alpha=12, \ldots, n$, is an almost para-Nordenian manifold.

Proof. If we put

$$
A(\tilde{X}, \tilde{Y})={ }^{S} g\left(F_{\alpha} \tilde{X}, \tilde{Y}\right)-{ }^{S} g\left(\tilde{X}, F_{\alpha} \tilde{Y}\right)
$$

for any $\tilde{X}, \tilde{Y} \in \Im_{0}^{1}\left(F^{*}(M)\right)$. Then from (3.1)-(3.3) and (4.1), we have

$$
\begin{gathered}
A\left({ }^{H} X,{ }^{H} Y\right)={ }^{S} g\left(F_{\alpha}\left({ }^{H} X\right),{ }^{H} Y\right)-{ }^{S} g\left({ }^{H} X, F_{\alpha}\left({ }^{H} Y\right)\right) \\
={ }^{S} g\left({ }^{V_{\alpha}} \tilde{X},{ }^{H} Y\right)-{ }^{S} g\left({ }^{H} X,{ }^{V_{\alpha}} \tilde{Y}\right)=0, \\
A\left({ }^{H} X,{ }^{V_{\beta}} \omega\right)={ }^{S} g\left(F_{\alpha}\left({ }^{H} X\right),{ }^{V_{\beta}} \omega\right)-{ }^{S} g\left({ }^{H} X, F_{\alpha}\left({ }^{V_{\beta}} \omega\right)\right) \\
={ }^{S} g\left({ }^{V_{\alpha}} \tilde{X},{ }^{V_{\beta}} \omega\right)-{ }^{S} g\left({ }^{H} X, \delta_{\alpha}^{\beta H} \tilde{\omega}\right) \\
\delta^{\alpha \beta} g{ }^{-1}(g X, \omega)-g\left(X, g^{-1} \omega\right) \delta_{\alpha}^{\beta}=0, \\
A\left({ }^{V_{\beta}} \omega,{ }^{H} Y\right)=-A\left({ }^{H} Y,{ }^{V_{\beta}} \omega\right)=0, \\
A\left({ }^{V_{\beta}} \omega,{ }^{V \gamma} \theta\right)={ }^{S} g\left(F_{\alpha}\left({ }^{V_{\beta}} \omega\right),{ }^{V \gamma} \theta\right)-{ }^{S} g\left({ }^{V_{\beta}} \omega, F_{\alpha}\left({ }^{V \gamma} \theta\right)\right) \\
={ }^{S} g\left(\delta_{\alpha}^{\beta H} \tilde{\omega},{ }^{V \gamma} \theta\right)-{ }^{S} g\left({ }^{V_{\beta}} \omega, \delta_{\alpha}^{\gamma H} \tilde{\theta}\right) \\
=\delta_{\alpha}^{\beta S} g\left({ }^{H} \tilde{\omega},{ }^{V \gamma} \theta\right)-\delta_{\alpha}^{\gamma S} g\left({ }^{V_{\beta}} \omega,{ }^{H} \tilde{\theta}\right)=0,
\end{gathered}
$$

i.e. ${ }^{S} g$ is pure with respect to $F_{\alpha}$, for each $\alpha=12, \ldots, n$. Thus Theorem 4.1 is proved. 
Let us consider the covariant derivative of $F_{\alpha}$, for each $\alpha=12, \ldots, n$, with respect to the Levi-Civita connection ${ }^{S} \nabla$ of metric ${ }^{S} g$. Taking into account $(i)-(i v)$ of Theorem 3.1, we obtain

$$
\begin{aligned}
& \left({ }^{S} \nabla_{H_{X}} F_{\alpha}\right)\left({ }^{H} Y\right)={ }^{H} \nabla_{H_{X}} F_{\alpha}\left({ }^{H} Y\right)-F_{\alpha}\left({ }^{S} \nabla_{H_{X}}{ }^{H} Y\right) \\
& ={ }^{S} \nabla_{H_{X}}{ }^{V_{\alpha}} \tilde{Y}-F_{\alpha}\left({ }^{H}\left(\nabla_{X} Y\right)+\frac{1}{2} \sum_{\beta=1}^{n} V_{\beta}\left(X^{\beta} \circ R(X, Y)\right)\right) \\
& =V_{\alpha}\left(\nabla_{X} \tilde{Y}\right)+\frac{1}{2}\left(R\left(\tilde{X}^{\alpha}, \tilde{\tilde{Y}}\right) X\right)-F_{\alpha}\left({ }^{H}\left(\nabla_{X} Y\right)+\frac{1}{2} \sum_{\beta=1}^{n} V_{\beta}\left(X^{\beta} \circ R(X, Y)\right)\right) \\
& =\frac{1}{2} H\left(R\left(\tilde{X}^{\alpha}, Y\right) X\right)-\frac{1}{2} \delta_{\alpha}^{\beta} \sum_{\beta=1}^{n}{ }^{H}\left(g^{-1} X^{\beta} \circ R(X, Y)\right) \\
& =\frac{1}{2} H\left(g^{-1} X^{\alpha}(R(\quad, Y) X-R(X, Y))\right), \\
& \left({ }^{S} \nabla_{V_{\beta} \omega} F_{\alpha}\right)\left({ }^{H} Y\right)={ }^{H} \nabla_{V_{\beta} \omega} F_{\alpha}\left({ }^{H} Y\right)-F_{\alpha}\left({ }^{S} \nabla_{V_{\beta} \omega}{ }^{H} Y\right) \\
& ={ }^{S} \nabla_{V_{\beta} \omega}{ }^{V_{\alpha}} \tilde{Y}-\frac{1}{2} F_{\alpha}{ }^{H}\left(R\left(\tilde{X}^{\beta}, \tilde{\omega}\right) Y\right)=-\frac{1}{2} F_{\alpha}{ }^{H}\left(X^{\beta} g^{-1} \circ R(, \tilde{\omega}) Y\right) \\
& =-\frac{1}{2} V_{\alpha}\left(X^{\beta} \circ R(, \tilde{\omega}) Y\right), \\
& \left({ }^{S} \nabla_{H_{X}} F_{\alpha}\right)\left({ }^{V_{\gamma}} \theta\right)={ }^{H} \nabla_{H_{X}} F_{\alpha}\left({ }^{V_{\gamma}} \theta\right)-F_{\alpha}\left({ }^{S} \nabla_{H_{X}}{ }^{V_{\gamma}} \theta\right) \\
& ={ }^{S} \nabla_{H_{X}} \delta_{\alpha}^{\gamma H} \tilde{\theta}-F_{\alpha}\left({ }^{V_{\gamma}}\left(\nabla_{X} \theta\right)+{ }^{1}{ }^{H}\left(R\left(\tilde{X}^{\gamma}, \tilde{\theta}\right) X\right)\right) \\
& =\delta_{\alpha}^{\gamma H}\left(\nabla_{X} \tilde{\theta}\right)+\delta_{\alpha}^{\gamma} \frac{1}{2} \sum_{\beta=1}^{n} V_{\beta}\left(X^{\beta} \circ R(X, \tilde{\theta})\right)-\delta_{\alpha}^{\gamma H}\left(g^{-1} \circ \nabla_{X} \theta\right) \\
& -\frac{1}{2} V_{\alpha}\left(g \circ R\left(\tilde{X}^{\gamma}, \tilde{\theta}\right) X\right)=\delta_{\alpha}^{\gamma} \frac{1}{2} \sum_{\beta=1}^{n} V_{\beta}\left(X^{\beta} \circ R(X, \tilde{\theta})\right) \\
& -\frac{1}{2} V_{\alpha}\left(g \circ R\left(\tilde{X}^{\gamma}, \tilde{\theta}\right) X\right), \\
& \left({ }^{S} \nabla_{V_{\beta} \omega} F_{\alpha}\right)\left({ }^{V_{\gamma}} \theta\right)={ }^{H} \nabla_{V_{\beta} \omega} F_{\alpha}\left({ }^{V_{\gamma}} \theta\right)-F_{\alpha}\left({ }^{S} \nabla_{V_{\beta} \omega}{ }^{V_{\gamma}} \theta\right) \\
& ={ }^{S} \nabla_{V_{\beta} \omega}\left(\delta_{\alpha}^{\gamma H} \tilde{\theta}\right)=\delta_{\alpha}^{\gamma S} \nabla_{V_{\beta} \omega}{ }^{H} \tilde{\theta}=\delta_{\alpha}^{\gamma} \frac{1}{2}{ }^{H}\left(R\left(\tilde{X}^{\beta}, \tilde{\omega}\right) \tilde{\theta}\right) \\
& =\delta_{\alpha}^{\gamma} \frac{1}{2} H\left(X^{\beta} g^{-1} \circ R(, \tilde{\omega}) \tilde{\theta}\right) .
\end{aligned}
$$

From (4.2)-(4.5), we get

Theorem 4.2. The linear coframe bundle $F^{*}(M)$ of a Riemannian manifold $(M, g)$ is para-Kahlerian (para-holomorphic Nordenian) with respect to the metric ${ }^{S} g$ and almost para-complex structure $F_{\alpha}$, for each $\alpha=12, \ldots, n$, if and only if the Riemannian manifold $(M, g)$ is flat. 


\section{Para-holomorphic vector-fields on $\left.\left(F^{*}(M),{ }^{S} g\right)\right)$}

Let $(M, g)$ be a Riemannian manifold, and let $F^{*}(M)$ be its linear coframe bundle with Sasakian metric ${ }^{S} g$ and with the almost para-Nordenian structures $F_{\alpha}$, $\alpha=1,2, \ldots, n$. A vector field $\tilde{X} \in \Im_{0}^{1}\left(F^{*}(M)\right)$ with respect to which the almost para-Nordenian structure $F_{\alpha}$ has a vanishing Lie derivative $\left(L_{\tilde{X}} F_{\alpha}=0\right)$ is said to be almost para-holomorphic (see, [18]). It is well known that [5]

$$
\begin{gathered}
{\left[{ }^{C} X,{ }^{H} Y\right]={ }^{H}[X, Y]+\sum_{\alpha=1}^{n} V_{\alpha}\left(X^{\alpha} \circ\left(L_{X} \nabla\right) Y\right),} \\
{\left[{ }^{C} V,{ }^{V_{\gamma}} \omega\right]={ }^{V_{\gamma}}\left(L_{V} \omega\right)}
\end{gathered}
$$

for any $X, Y \in \Im_{0}^{1}(M)$ and $\omega \in \Im_{1}^{0}(M)$, where

$$
\left(L_{X} \nabla\right) Y=\nabla_{Y} \nabla X+R(X, Y)
$$

and

$$
\left(L_{X} \nabla\right)(Y, Z)=L_{X}\left(\nabla_{Y} X\right)-\nabla_{Y}\left(L_{X} Z\right)-\nabla_{[X, Y]} Z
$$

A vector field $X \in \Im_{0}^{1}(M)$ is called a Killing vector field (or infinitesimal isometry) if $L_{X} g=0$ and $X$ called an infinitesimal affine transformation if $L_{V} \nabla g=0$. A Killing vector field is necessarily an infinitesimal affine transformation, i.e. we have $L_{V} \nabla g=0$ as a consequence of $L_{X} g=0$. Now we consider the Lie derivative of $F_{\alpha}(\alpha=1,2, \ldots, n)$ with respect to the complete lift ${ }^{C} X$. Taking account of (4.1), (5.1) and (5.2), we find

$$
\begin{gathered}
\left(L_{C_{X}} F_{\alpha}\right)\left({ }^{V_{\gamma}} \theta\right)=L_{C_{X}}\left(F_{\alpha}\left({ }^{V_{\gamma}} \theta\right)-F_{\alpha}\left(L_{X}{ }^{V_{\gamma}} \theta\right)\right. \\
=\delta_{\alpha}^{\gamma} L_{C_{X}}{ }^{H} \tilde{\theta}-\delta_{\alpha}^{\gamma}\left(g^{-1} \circ\left(L_{X} \theta\right)\right)=\delta_{\alpha}^{\gamma}\left[{ }^{H}[X, \tilde{\theta}]+\sum_{\beta=1}^{n} V_{\beta}\left(X^{\beta} \circ L_{X} \nabla \tilde{\theta}\right)\right. \\
\left.-{ }^{H}\left(g^{-1} \circ\left(L_{X} \theta\right)\right)\right]=\delta_{\alpha}^{\gamma}\left[{ }^{H}\left(L_{X}\left(g^{-1} \circ \theta\right)-g^{-1} \circ\left(L_{X} \theta\right)\right)\right. \\
\left.+\sum_{\beta=1}^{n}{ }^{V_{\beta}}\left(X^{\beta} \circ L_{X} \nabla \tilde{\theta}\right)\right] \\
\left(L_{C}{ } F_{\alpha}\right)\left({ }^{H} Y\right)=L_{C}\left(F_{\alpha}\left({ }^{H} Y\right)-F_{\alpha}\left(L_{X}{ }^{H} Y\right)\right. \\
=L_{C}{ }^{V_{\alpha}} \tilde{Y}-F_{\alpha}\left({ }^{H}[X, Y]+\sum_{\beta=1}^{n}{ }^{V_{\beta}}\left(X^{\beta} \circ L_{X} \nabla Y\right)\right. \\
=L_{C}{ }^{V_{\alpha}} \tilde{Y}-{ }^{V_{\alpha}}(g \circ[X, Y])+\sum_{\beta=1}^{n} \delta_{\alpha}^{\beta V_{\beta}}\left(g^{-1} X^{\beta} \circ L_{X} \nabla Y\right) \\
={ }^{V_{\alpha}}\left(L_{X}(g \circ Y)-g \circ L_{X} Y\right)+\sum_{\beta=1}^{n} \delta_{\alpha}^{\beta V_{\beta}}\left(g^{-1} X^{\beta} \circ L_{X} \nabla Y\right) .
\end{gathered}
$$

Let now $X$ be a Killing vector field $\left(L_{X} g=0\right)$. Then by virtue of $L_{X} \nabla=0$, from (5.3) and (5.4), we have $L_{C}{ }_{X} F_{\alpha}=0, \alpha=1,2, \ldots, n$, i.e. ${ }^{C} X$ is paraholomorphic with respect to each $F_{\alpha}$. Hence, we have 
Theorem 5.1. Let $(M, g)$ be a Riemannian manifold and let $\left(F^{*}(M),{ }^{S} g, F_{\alpha}\right)$ be para-Nordenian manifold for each $\alpha=1,2, \ldots, n$. Then the complete lift ${ }^{C} X$ of vector field $X \in \Im_{0}^{1}(M)$ to $F^{*}(M)$ is almost para-holomorphic vector field with respect to the each almost para-Nordenian structure $\left(F_{\alpha},{ }^{S} g\right)$, if $X$ is a Killing vector field on a Riemannian manifold $(M, g)$.

\section{Integrability of para-Nordenian structures on $\left.\left(F^{*}(M),{ }^{S} g\right)\right)$}

In this section, we study the integrability of almost para-Nordenian structure $F_{\alpha}$ for each $\alpha=1,2, \ldots, n$, on $\left.\left(F^{*}(M),{ }^{S} g\right)\right)$. We assume that $\nabla$ is a Levi-Civita connection of a Riemannian metric $g$. Denoting by $N_{F_{\alpha}}$ the Nijenhuis tensor of $F_{\alpha}$, we have

$$
N_{F_{\alpha}}(\tilde{X}, \tilde{Y})=\left[F_{\alpha} \tilde{X}, F_{\alpha} \tilde{Y}\right]-F_{\alpha}\left[F_{\alpha} \tilde{X}, \tilde{Y}\right]-F_{\alpha}\left[\tilde{X}, F_{\alpha} \tilde{Y}\right]+[\tilde{X}, \tilde{Y}]
$$

for all $\tilde{X}, \tilde{Y} \in \Im_{0}^{1}\left(F^{*}(M)\right)$. Then taking account of (2.6) and (6.1), we obtain

$$
\begin{aligned}
& N_{F_{\alpha}}\left({ }^{H} X,{ }^{H} Y\right)=\left[F_{\alpha}{ }^{H} X, F_{\alpha}{ }^{H} Y\right]-F_{\alpha}\left[F_{\alpha}{ }^{H} X,{ }^{H} Y\right] \\
& -F_{\alpha}\left[{ }^{H} X, F_{\alpha}{ }^{H} Y\right]+\left[{ }^{H} X,{ }^{H} Y\right]=\left[{ }^{V_{\alpha}} \tilde{X},{ }^{V_{\alpha}} \tilde{Y}\right]-F_{\alpha}\left[{ }^{V_{\alpha}} \tilde{X},{ }^{H} Y\right] \\
& -F_{\alpha}\left[{ }^{H} X,{ }^{V_{\alpha}} \tilde{Y}\right]+{ }^{H}[X, Y]+\sum_{\beta=1}^{n} V_{\beta}\left(X^{\beta} \circ R(X, Y)\right) \\
& =F_{\alpha}{ }^{V_{\alpha}}\left(\nabla_{Y} \tilde{X}\right)-F_{\alpha}{ }^{V_{\alpha}}\left(\nabla_{X} \tilde{Y}\right)+{ }^{H}[X, Y]+\sum_{\beta=1}^{n} V_{\beta}\left(X^{\beta} \circ R(X, Y)\right) \\
& =\delta_{\alpha}^{\alpha H}\left(\nabla_{Y} X-\nabla_{X} Y\right)+{ }^{H}[X, Y]+\sum_{\beta=1}^{n} V_{\beta}\left(X^{\beta} \circ R(X, Y)\right) \\
& =\sum_{\beta=1}^{n} V_{\beta}\left(X^{\beta} \circ R(X, Y)\right), \\
& N_{F_{\alpha}}\left({ }^{H} X,{ }^{V_{\beta}} \omega\right)=\left[F_{\alpha}{ }^{H} X, F_{\alpha}{ }^{V_{\beta}} \omega\right]-F_{\alpha}\left[F_{\alpha}{ }^{H} X,{ }^{V_{\beta}} \omega\right] \\
& -F_{\alpha}\left[{ }^{H} X, F_{\alpha}{ }^{V_{\beta}} \omega\right]+\left[{ }^{H} X,{ }^{V_{\beta}} \omega\right]=\left[{ }^{V_{\alpha}} \tilde{X}, \delta_{\alpha}^{\beta H} \tilde{\omega}\right]-F_{\alpha}\left[{ }^{V_{\alpha}} \tilde{X},{ }^{V_{\beta}} \omega\right] \\
& -F_{\alpha}\left[{ }^{H} X, \delta_{\alpha}^{\beta H} \tilde{\omega}\right]+{ }^{V_{\beta}}\left(\nabla_{X} \omega\right)=\delta_{\alpha}^{\beta}\left[{ }^{V_{\alpha}} \tilde{X},{ }^{H} \tilde{\omega}\right]+{ }^{V_{\beta}}\left(\nabla_{X} \omega\right) \\
& -\delta_{\alpha}^{\beta} F_{\alpha}\left({ }^{H}[X, \tilde{\omega}]+\sum_{\gamma=1}^{n} V_{\gamma}\left(X^{\gamma} \circ R(X, \tilde{\omega})\right)\right)=-\delta_{\alpha}^{\beta V_{\alpha}}\left(\nabla_{\tilde{\omega}} \tilde{X}\right) \\
& +{ }^{V_{\beta}}\left(\nabla_{X} \omega\right)-\delta_{\alpha}^{\beta V_{\alpha}}(g \circ[X, \tilde{\omega}])-\delta_{\alpha}^{\beta} \sum_{\gamma=1}^{n} \delta_{\alpha}^{\gamma H}\left(g^{-1} X^{\gamma} \circ R(X, \tilde{\omega})\right) \\
& =-{ }^{V_{\beta}}\left(g \circ \nabla_{\tilde{\omega}} X\right)+{ }^{V_{\beta}}\left(g \circ \nabla_{X} \tilde{\omega}\right)-{ }^{V_{\beta}}(g \circ[X, \tilde{\omega}]) \\
& -\delta_{\alpha}^{\beta} \sum_{\gamma=1}^{n} \delta_{\alpha}^{\gamma H}\left(g^{-1} X^{\gamma} \circ R(X, \tilde{\omega})\right)={ }^{V_{\beta}}(g \circ[X, \tilde{\omega}])-{ }^{V_{\beta}}(g \circ[X, \tilde{\omega}]) \\
& =-\delta_{\alpha}^{\beta} \sum_{\gamma=1}^{n} \delta_{\alpha}^{\gamma H}\left(g^{-1} X^{\gamma} \circ R(X, \tilde{\omega})\right), \\
& N_{F_{\alpha}}\left({ }^{V_{\beta}} \omega,{ }^{V_{\gamma}} \theta\right)=\left[F_{\alpha}{ }^{V_{\beta}} \omega, F_{\alpha}{ }^{V_{\gamma}} \theta\right]-F_{\alpha}\left[F_{\alpha}{ }^{V_{\beta}} \omega,{ }^{V_{\gamma}} \theta\right]
\end{aligned}
$$




$$
\begin{gathered}
-F_{\alpha}\left[{ }^{V_{\beta}} \omega, F_{\alpha}{ }^{V_{\gamma}} \theta\right]+\left[{ }^{V_{\beta}} \omega,{ }^{V_{\gamma}} \theta\right]=\left[\delta_{\alpha}^{\beta H} \tilde{\omega}, \delta_{\alpha}^{\gamma H} \tilde{\theta}\right] \\
\left.-F_{\alpha}\left[\delta_{\alpha}^{\beta H} \tilde{\omega},{ }^{V_{\gamma}} \theta\right]-F_{\alpha}{ }^{V_{\beta}} \omega, \delta_{\alpha}^{\gamma H} \tilde{\theta}\right]=\delta_{\alpha}^{\beta} \delta_{\alpha}^{\gamma}\left[{ }^{H} \tilde{\omega},{ }^{H} \tilde{\theta}\right] \\
-\delta_{\alpha}^{\beta} F_{\alpha}\left[{ }^{H} \tilde{\omega},{ }^{V_{\gamma}} \theta\right]-\delta_{\alpha}^{\gamma} F_{\alpha}\left[{ }^{V_{\beta}} \omega,{ }^{H} \tilde{\theta}\right]=\delta_{\alpha}^{\beta} \delta_{\alpha}^{\gamma H}[\tilde{\omega}, \tilde{\theta}] \\
+\delta_{\alpha}^{\beta} \delta_{\alpha}^{\gamma} \sum_{\sigma=1}^{n}{ }^{V_{\sigma}}\left(X^{\sigma} \circ R(\tilde{\omega}, \tilde{\theta})\right)-\delta_{\alpha}^{\beta} F_{\alpha}{ }^{V_{\gamma}}\left(\nabla_{\tilde{\omega}} \theta\right) \\
+\delta_{\alpha}^{\gamma} F_{\alpha}{ }^{V_{\beta}}\left(\nabla_{\tilde{\theta}} \omega\right)=\delta_{\alpha}^{\beta} \delta_{\alpha}^{\gamma H}[\tilde{\omega}, \tilde{\theta}]+\delta_{\alpha}^{\beta} \delta_{\alpha}^{\gamma} \sum_{\sigma=1}^{n}{ }^{V_{\sigma}}\left(X^{\sigma} \circ R(\tilde{\omega}, \tilde{\theta})\right) \\
-\delta_{\alpha}^{\beta} \delta_{\alpha}^{\gamma H}\left(g^{-1} \circ \nabla_{\tilde{\omega}} \theta\right)+\delta_{\alpha}^{\gamma} \delta_{\alpha}^{\beta H}\left(g^{-1} \circ \nabla_{\tilde{\theta}} \omega\right)=\delta_{\alpha}^{\beta} \delta_{\alpha}^{\gamma H}[\tilde{\omega}, \tilde{\theta}] \\
+\delta_{\alpha}^{\beta} \delta_{\alpha}^{\gamma} \sum_{\sigma=1}^{n} V_{\sigma}\left(X^{\sigma} \circ R(\tilde{\omega}, \tilde{\theta})\right)-\delta_{\alpha}^{\beta} \delta_{\alpha}^{\gamma H}\left(\nabla_{\tilde{\omega}} \tilde{\theta}\right) \\
+\delta_{\alpha}^{\gamma} \delta_{\alpha}^{\beta H}\left(\nabla_{\tilde{\theta}} \tilde{\omega}\right)=\delta_{\alpha}^{\beta} \delta_{\alpha}^{\gamma} \sum_{\sigma=1}^{n} V_{\sigma}\left(X^{\sigma} \circ R(\tilde{\omega}, \tilde{\theta})\right)
\end{gathered}
$$

for all $X, Y \in \Im_{0}^{1}(M), \omega, \theta \in \Im_{1}^{0}(M)$ and $1 \leq \alpha \leq n$.

From the above equations we conclude that $N_{F_{\alpha}}=0$ if and only if $R(X, Y)=0$ for all $X, Y \in \Im_{0}^{1}(M)$. Therefore, we have

Theorem 6.1. Let $(M, g)$ be a Riemannian manifold and $F^{*}(M)$ be its linear coframe bundle equipped with Sasakian metric ${ }^{S} g$. Then the almost paraNordenian manifold $\left(F^{*}(M),{ }^{S} g, F_{\alpha}\right)$, for each $\alpha=1,2, \ldots, n$, is para-Nordenian if and only if $R=0$.

\section{References}

[1] K. Aso, Notes on some properties of the sectional curvature of the tangent bundle, Yokohama Math. J. 29 (1981), 1-5.

[2] L.A.Cordero, C.T. Dodson and de M. Leon, Differential Geometry of Frame Bundles, Mathematics and its Applications, Kluwer Academic Publishers Group, Dordrecht 1989.

[3] L.A.Cordero and de M.Leon, On the curvature of the induced Riemannian metric on the frame bundle of a Riemannian manifold, J. Math. pures et appl. 65 (1986), 81-91.

[4] L. S. Druta, Classes of general natural almost anti-Hermitian structures on the cotangent bundles, Mediterr. J. Math. 8 (2011), no.2, 161-179,.

[5] H.D.Fattayev and A.A.Salimov, Diagonal lifts of metrics to coframe bundle,Proc. of IMM of NAS of Azerbaijan 44 (2018), no.2, 328-337.

[6] U.K.Gautam, Abdul Haseeb and Rajendra Prasad, Some results on projective curvature tensor in Sasakian manifolds, Commun. Korean Math. Soc. 34 (2019), no.3, 881-896.

[7] V.A.Khan and M.A.Khan, Pseudo-slant submanifolds of a Sasakian manifold, Indian J. Pure Appl. Math. 38 (2007), no.1, 31-42.

[8] O.Kowalski, Curvature of the induced Riemannian metric on the tangent bundle of Riemannian manifold, J. reine angew Math.,250 (1971), 124-129.

[9] O.Kowalski and M.Sekizawa, On curvatures of linear frame bundle with naturally lifted metrics, Rend. Sem. Mat. Univ. Pol. Torino, 63(2005), no.3, 283-296.

[10] K. P. Mok, Metrics and connections on the cotangent bundle, Kodai Math. Sem. Rep. 28 (1977), 226-238. 
[11] E.Musso and F.Tricerri, Riemannian metrics on tangent bundles, Ann. Mat. Pura Appl. 150 (1988), no.4, 1-20.

[12] Z.Olszak, On almost complex structures with Norden metrics on tangent bundles, Period. Math. Hungar. 51 (2005), no.2, 59-74.

[13] V.Oproiu and N. Papaghiuc, Some classes of almost anti-Hermitian structures on the tangent bundle, Mediterr. J. Math.,1 (2004), no.3, 269-282.

[14] E.Peyghan, H.Nasrabadi and A.Tayebi, The homogeneous lift to the (1,1)-tensor bundle of a Riemannian metric, Int. J. of Geom. Meth. In Mod. Phys. 10 (2013), no.4, 1-18.

[15] A.Salimov, A.Gezer and M. Iscan, On para-Kähler-Norden structures on the tangent bundles, Ann. Polon. Math. 103 (2012), no.3, 247-261.

[16] A. Salimov and A.Gezer, On the geometry of the $(1,1)$-tensor bundle with Sasaki type metric, Chin. Ann. Math. Ser. B, 32 (2011), no.3, 369-386.

[17] A.A.Salimov, A.Gezer and S.Akbulut, Geodesics of Sasakian metrics on the tensor bundles, Mediterr. J. Math. 6 (2009), no.2, 137-149.

[18] A.A.Salimov and F.Agca, Some properties of Sasakian metrics in cotangent bundles, Mediterr. J. Math. 8 (2011), 243-255.

[19] A. A. Salimov and H.D. Fattayev, Connections On The Coframe Bundle, Inter. Elect. J. of Geom. 12 (2019), no.1, 93-101.

[20] A.A.Salimov and H.D.Fattaev, Coframe bundle and problems of lifts on its crosssections, Turk J Math. 42 (2018), no.4, 2035-2044.

[21] S.Sasaki, On the differential geometry of tangent bundles of Riemannian manifolds, Tohoku Math. J. 10 (1958), 338-354.

[22] K.Yano and S.Ishihara, Tangent and cotangent bundles, Marsel Dekker, Inc., New York 1973.

\section{Habil Fattayev}

Baku State University, 23, Z.Khalilov str., AZ1148, Baku, Azerbaijan

E-mail address: h-fattayev@mail.ru

Received: June 1, 2020; Revised: December 4, 2020; Accepted: December 27, 2020 